\title{
Keine Parallelmilieus in Deutschland
}

\section{Was passiert, wenn der Sinus-Milieu-Ansatz auf die Bevölkerung mit Migrationshintergrund in Deutschland übertragen wird}

\author{
Thomas Kunz
}

\section{Prof. Dr. Thomas Kunz ist}

Vertretungsprofessor an der Fachhochschule Frankfurt am Main für das Fachgebiet "Soziale Arbeit in der Einwanderungsgesellschaft « E-Mailmtkunz@fb4.fh-frankfurt.de
Bei der Bevölkerungsgruppe der Menschen mit Migrationshintergrund können acht unterschiedliche Milieus unterschieden werden, stellt eine neue Studie fest. Die Konzeption der Untersuchung wie auch ibre Ergebnisse bedürfen einer kritischen Betrachtung.

Im Oktober 2007 wurden der Öffentlichkeit die Ergebnisse der Sinus-Studie »Die Milieus der Menschen mit Migrationshintergrund in Deutschland « präsentiert. (1) Hierbei handelt sich um eine qualitative sozialwissenschaftliche Studie, die von der Heidelberger Firma SINUS SOCIOVISION GmbH im Auftrag des Bundesministeriums für Familie, Senioren, Frauen und Jugend, der MW Malteser Werke gGmbH, der Staatskanzlei Nordrhein-Westfalen, der Landeshauptstadt München, dem Südwestrundfunk und dem vhw Bundesverband für Wohneigentum und Stadtentwicklung e.V. durchgeführt wurde. (2) Das Unterfangen, den aus der Marktforschung stammenden Sinus-Milieu-Ansatz nun auch mit Blick auf die Migrantenpopulation in Deutschland fruchtbar zu machen, birgt einige interessante Implikationen, wirft aber auch die Frage auf, ob solch eine »Parallelisierung « von Milieuwelten migrations- und integrationspolitisch womöglich kontraproduktiv ist. Soll heißen: Es geht an dieser Stelle nicht um die Frage des Ob, sondern des Wie in Bezug auf die Übertragung des Milieu-Ansatzes.

Der Sinus-Milieu-Ansatz findet in der Marktforschung bereits seit längerem Anwendung. Er wird als ein Instrument für strategisches Marketing und als Tool für die Optimierung von Media-Zielgruppen verstanden. Er verortet Milieus einerseits nach sozialer Lage in Schichten, auf der Grundlage von Bildung, Beruf und Einkommen. Zum anderen nach Grundorientierungen in einem Spannungsbogen von traditionell bis postmodern. Die bisherigen, migrationsunspezifischen SinusMilieus werden in einer charakteristi- schen Form visualisiert, die wegen ihrer typischen Darstellung bis hinein in Fachkreise als sogenannte "Kartoffelgrafik « bekannt ist. Der Sinus-Milieu-Ansatz ist ein markenrechtlich geschütztes Produkt der SINUS SOCIOVISION GmbH. Der Schutz bedeutet zugleich, dass der Ansatz en detail nicht zugänglich ist, eine sozialwissenschaftlich Weiterbearbeitung der Ergebnisse, also eine im Wortsinne unabhängige Nach-Forschung nicht möglich ist. Die Studie verweist indes durchaus auf migrationssoziologische Desiderata und es wäre durchaus spannend, dem Ansatz und seiner Tragfähigkeit mittels vertiefender und variierter Milieustudien weiter nachzugehen.

Die nun vorgelegte Studie bedeutet die Übertragung des Sinus-Milieu-Ansatzes auf die Untersuchung der Gruppe der Menschen mit Migrationshintergrund in Deutschland. Dies ist ein Novum. Das Schichtenmodell und das Spektrum der Grundorientierungen wurden hierzu wenn auch modifiziert - für die Beschreibung von Milieus der Migrantinnen und Migranten in Deutschland beibehalten. Gleiches gilt für deren Visualisierung (siehe Abbildung Seite 131). Es ist insofern naheliegend, von einer »Kartoffelgrafik mit Migrationshintergrund « zu sprechen.

Wohl in Anerkenntnis der Schwierigkeit, nicht einfach eine Schichtzuordnung nach altem Vorbild vornehmen zu können, wurde hinsichtlich der Migrantinnen und Migranten, deren soziale Lage lediglich als niedrig, mittel und hoch klassifiziert. Demgegenüber unterscheidet die klassische Darstellung, also die Milieulandschaft »ohne Migrationshintergrund «, nach Unter-, Mittel- und Oberschichtzugehörigkeit. (3) Auch die Grundorientierungen wurden diversifiziert und interkulturell bzw. ethnisch »angereichert «. So gliedert sich die traditionelle Orientierung in der neuen Studie beispielsweise in eine sogenannte " archaische Tradition « einerseits und eine »ethnische Tradition« andererseits. Auch in 
den anderen Orientierungsmustern finden sich Hinweise, die auf die gedachte Spezifik der untersuchten Klientel abzielen, wie beispielsweise auf »bi-kulturelle Orientierung « oder » multikulturelle Identifikation «.

In der beschriebenen Exklusivität der Geltung kultureller Zugehörigkeit liegt einer der Kritikpunkte an besagter Studie begründet: Hierdurch werden die Milieus quasi kulturalisiert, es wird also die Gültigkeit der Dimension Migrationshintergrund mittels dem Versuch besonders betont, die Frage zu beantworten, inwieweit zwischen Milieubildungen und Migrationshintergrund ein Zusammenhang bestehen könnte.

Die quantitative Grundlage, auf der die Befunde erhoben wurden, ist als relativ dünn anzusehen. Zwischen Oktober 2006 und Mai 2007 wurden circa 100 mehrstündige Tiefeninterviews mit $\mathrm{Mi}$ grantinnen und Migranten unterschiedlicher ethnischer Herkunft, Alter, Geschlecht und Bildung durchgeführt und sozialwissenschaftlich ausgewertet. Gleichwohl eine Basis, die ausreicht, um mittels explorativem Vorgehen zur ermitteln, welche Erkenntnisse die Übertragung des Milieuansatzes zutage fördert. Folgende Ergebnisse werden unter anderem besonders hervorgehoben (4):

- Auf Basis der Erhebungen werden von den Forschern in der Gruppe der Menschen mit Migrationshintergrund acht unterschiedliche Milieus, die untereinander Überschneidungen aufweisen, verortet: religiös-verwurzeltes Milieu, traditionelles Gastarbeitermilieu, statusorientiertes Milieu, entwurzeltes Milieu, intellektuell-kosmopolitisches Milieu, adaptives Integrationsmilieu, multikulturelles Performermilieu und ein hedonistisch-subkulturelles Milieu.

- Die Migranten-Population in Deutschland erweise sich demnach als sehr facettenreich. Negativ-Klischees über Einwanderer würden durch die Studie zudem widerlegt.

- Die vorfindbaren Milieus unterscheiden sich, so eine weitere Erkenntnis, weniger nach ethnischer Herkunft und sozialer Lage als viel mehr nach ihren Wertvorstellungen, Lebensstilen und ästhetischen Vorlieben. Es bestünden Milieu-Bildungen und Milieu-Überschneidungen über unterschiedliche Herkunftskulturen hinweg. Milieubedingte Bindekräfte seien stärker als herkunftsspezifische.
- Zugleich zeige sich, dass Rückschlüsse von Herkunftskultur auf das Milieu und umgekehrt - nicht möglich seien. Ethnische Zugehörigkeit, Religion und Zuwanderungsgeschichte hätten zwar Einfluss auf die Alltagskultur, seien letztlich jedoch nicht milieuprägend und identitätsstiftend. Insbesondere der Einfluss religiöser Traditionen werde oft überschätzt.

- Die eingangs erwähnte Heterogenität der Migrantenpopulation werde auch in Bezug auf das Integrationsverhalten deutlich: Es werden - je nach Milieu unterschiedliche Abstufungen zwischen Assimilationstendenzen und Integrationsverweigerung identifiziert. Zugleich betonen die Forscher, dass die Milieus mehrheitlich aber um Integration bemüht seien.

- Die Auswertungen der Interviews zeigen darüber hinaus, dass die meisten Befragten sich, unter Beibehaltung ihrer kulturellen Wurzeln, in die Aufnahmegesellschaft einfügen wollen. Viele, vor allem jüngere Interviewpartner der zweiten und dritten Generation verfügen über ein bi-kulturelles Selbstbewusstsein und betrachten Migrationshintergrund und Mehrsprachigkeit als Bereicherung.

- Der Integrationsgrad sei wesentlich bildungs- und herkunftsabhängig.

- Quer durch die Migrantenmilieus werde zudem eine mangelnde Integrationsbereitschaft der Aufnahmegesellschaft beklagt.

Inwieweit dieser Erkenntnisertrag eher der Methode, also dem Einsatz mehrstündiger Tiefeninterviews zuzuschreiben ist und weniger dem Milieuansatz kann an dieser Stelle nicht vertieft werden. Die Vermutung liegt indes nahe. Es ist zu erwähnen, dass die Erkenntnis über Heterogenität und Differenziertheit der Migrantenpopulation in Deutschland und die bestehende Gefahr homogenisierender Annahmen über »die Migrantinnen und Migranten « - eigentlich bereits seit längerem zum State of the Art aufgeklärter migrationssoziologischer wie integrationspolitischer Sichtweisen gehört. Auf diesen Sachverhalt erneut und öffentlichkeitswirksam aufmerksam $\mathrm{zu}$ machen, ist indes durchaus ein Verdienst.

Wie erwähnt, identifizieren die SinusForscher acht migrantenspezifische Milieus. Im Vergleich zu den zehn Milieus in Deutschland, die im Rahmen der Sinus-
Studien bislang ohne die exponierte Berücksichtigung des Migrationshintergrundes identifiziert wurden, fällt also auf, dass zwei Milieus fehlen: Bei den Migrantenmilieus fehlen die sogenannten »Konservativen « und die »Etablierten «. Dies ist wenig verwunderlich, handelt es sich doch bei diesen um eher traditionelle Oberschichtmilieus, die allein aufgrund historischer Gegebenheiten im Einwanderungsland Deutschland für Migranten nicht existieren: ein traditionelles migrantisches Oberschichtmilieu kann es in diesem Land (noch) nicht geben, weil das Migrationsgeschehen im Zeitverlauf hierfür noch gar nicht lange genug andauert, um solch eine Schicht herausbilden zu können.

Ferner ist nochmals zu betonen, dass es sich lediglich um eine qualitative, nichtrepräsentative Studie handelt, die zahlenmäßigen Anteile der verorteten Milieus innerhalb der Gruppe der Menschen mit Migrationshintergrund sind also bislang unbekannt. Auf die Diskrepanz zwischen der zugrundeliegenden Zahl von 100 Interviews angesichts einer Zahl von über 15 Millionen Menschen mit Migrationshintergrund in Deutschland wurde hingewiesen. Diese Einschränkung anerkennen die Sinus-Forscher und sie dokumentiert sich in dem selbst geäußerten Hinweis, nach dem eine Quantifizierung des Modells noch ausstehe, um die Milieus zu validieren und deren eigentliche Größe und Struktur zu bestimmen. Dies bedeutet aber auch, dass Aussagen und Bewertungen, die im Zuge der öffentlichen Diskussion unter Heranziehung der Studie getroffen werden, unter Vorbehalt stehen. Schlussfolgerungen in Bezug auf die zahlenmäßigen Anteile und Gewichtungen, welche bestimmten Milieus in der Gruppe der Menschen mit Migrationshintergrund haben, sind vorsichtig zu handhaben und besitzen allenfalls vorläufigen Charakter.

\section{Bedeutung der Religion}

Mit Blick auf die Bedeutung, die in der öffentlichen Debatte um Migration und Integration der Religiosität beigemessen wird, erscheint es interessant, sich die Beschreibung des »religiös-verwurzelten Milieus « genauer anzuschauen. Laut Sinus-Studie sei dieses Migrantenmilieu insbesondere durch archaische Tradition ge- 
prägt. Die Forscher charakterisieren dessen Grundorientierung »AI Archaische Tradition « mittels Hinweisen auf die Geltung »traditioneller Werte « und »religiösen Dogmatismus«. Es verfüge darüber hinaus über ein "patriarchalisches Weltbild, überkommene Familienwerte und Zwangsnormen«. Dieses Milieu präge ferner ein »rigide-konventionalistischer Lebensstil « sowie »strenge Moral«. Wenig verwunderlich, dass hier kulturelle Enklavenbildung und nicht bloß mangelnde, sondern gleich »keine Integrationsbereitschaft « konstatiert werden.

Nun kommt man nachvollziehbarerweise nicht umhin, in einer Studie zum Thema Migrantenmilieus, auch Tendenzen und Dynamiken aufzugreifen und zu operationalisieren, die auf Hindernisse und Erschwernisse im Integrationsverlauf abheben. Soweit ist den Marktforschern durchaus zu folgen. Integration mittels der Klassifizierung von Bereitschaft vornehmlich als voluntaristischen Akt erscheinen zu lassen, der den oder die Einzelne sortiert in willig und unwillig und somit einen mangelnden Grad an gesellschaftlicher Integration als Ergebnis von Widerstand fasst, ist allerdings eine fragwürdige Reduk- tion, die eher als Klischee- denn als Milieubildung anmutet.

Religiosität und hier, also bei der Grundorientierung »AI Archaische Tradition «, deren Konkretisierung mittels der Formulierung »oft: islamisch « lassen Religiosität nicht bloß als Integrationshemmnis erscheinen, sondern implizieren insbesondere islamische Religionszugehörigkeit als Attribut, das auf Integrationsverweigerung hindeutet. Es verwundert folglich nicht, dass besagte Grundorientierung mittels dem Schlagwort »Parallelkultur « zugespitzt wird - die Rede von der Parallelgesellschaft lässt grüßen. Insofern kann die Studie durchaus als unausgewogen und stereotyp gedeutet werden. Zumal sich ähnlich exponierte Hinweise auf islamische Religionszugehörigkeit in keiner der anderen Grundorientierungen finden lassen.

Die Grundorientierung des religiös-verwurzelten Milieus konterkariert die ansonsten quer durch die anderen Migrantenmilieus konstatierte Klage über eine mangelnde Integrationsbereitschaft der Aufnahmegesellschaft - hier ist es umgekehrt. Im Milieu der religiös Verwurzelten werden quasi die Schmuddelkinder der Zuwanderungsgesellschaft entsorgt.
Fast schon erleichtert darf man zur Kenntnis nehmen, dass dieses Milieu nicht nur im Schaubild als randständig und entsprechend seiner geringen Größenanmutung nach vernachlässigbar ist: »Während negativ besetzte Themen wie islamischer Terror, Zwangsheirat und Frauen mit Kopftuch die Schlagzeilen beherrschen, zeigt die Studie, dass nur ein kleineres von insgesamt acht Milieus durch seine religiöse Bindung geprägt ist. Nur bei einer Minderheit der in Deutschland lebenden Einwanderer prägen also religiöse Traditionen den Alltag. «(Beck, Perry 2007: 192)

Abschließend $\mathrm{zu}$ diesem Gedankengang gilt es zu betonen, dass der hier geäußerte Kritikpunkt nicht darauf abzielt, integrationspolitische Aspekte, die im Kontext von Religion und Religionszugehörigkeit beobachtbar sein mögen, zu negieren. Jedoch geht es darum anzuerkennen, dass die Auswirkungen von Religionszugehörigkeit (insbesondere und gerade auch einer islamischen) vielfältiger sind, als es diese Typenbildung nahelegt und vor allem auch, dass eine solche nicht zwingend als Hinweis auf ein Integrationshemmnis oder Integrationswiderstand zu deuten ist.

\section{Die Migranten-Milieus in Deutschland 2007 Soziale Lage und Grundorientierung}

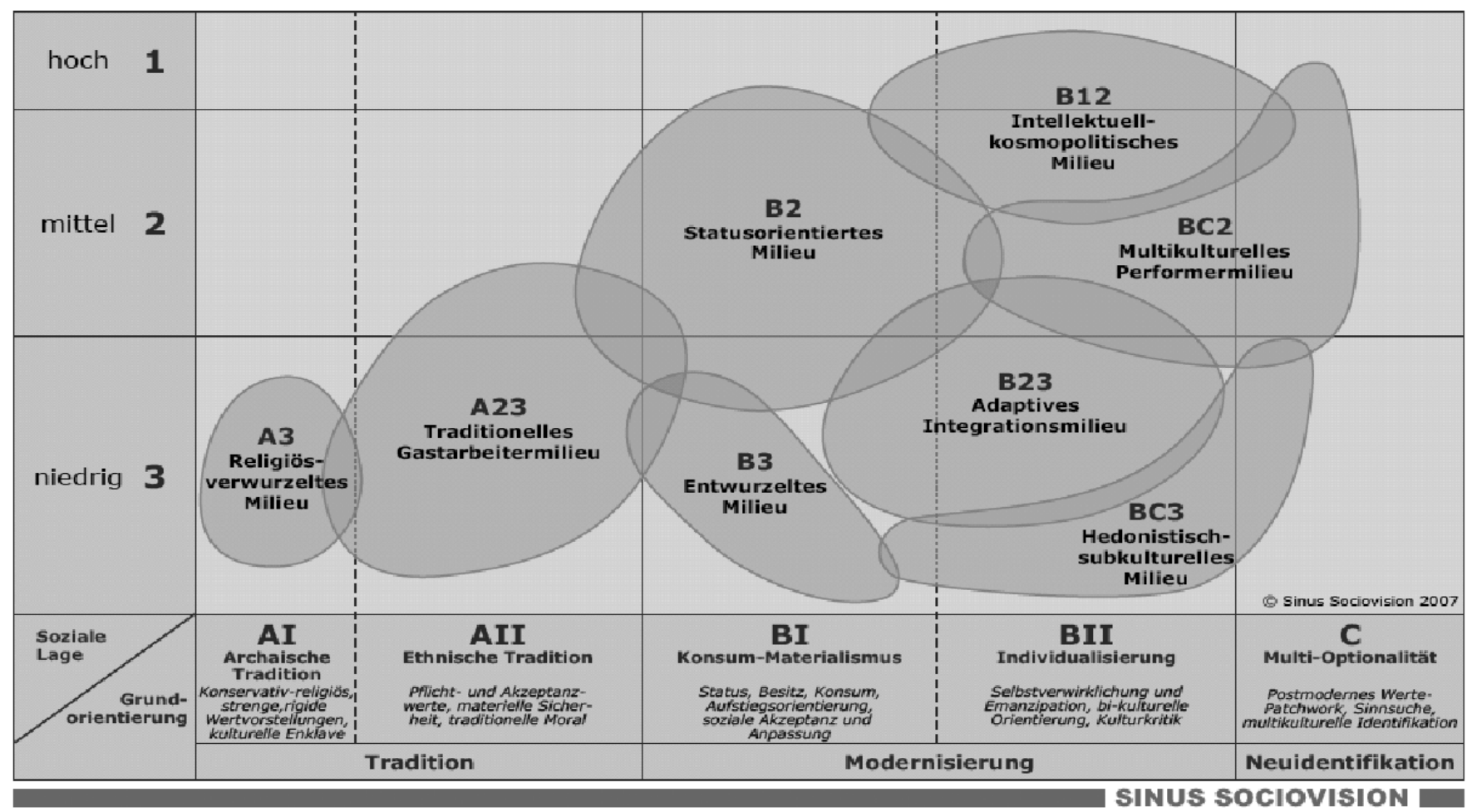

Quelle: Sociovision, 2007 


\section{Folgerungen}

Aus integrationspolitischer Sicht ist die Studie trotz der erwähnten Einschränkungen in dreierlei Weise relevant und anknüpfungsfähig:

- Sie bekräftigt, erstens, die Notwendigkeit, die Bevölkerung mit Migrationshintergrund in Deutschland differenziert zu betrachten und dokumentiert einmal mehr deren soziokulturelle Heterogenität - diesmal in Gestalt unterschiedlicher Milieus.
Hier wäre entsprechend differenziert vorzugehen.

Auf diesen Einwand weist implizit auch das Erkenntnisinteresse eines der Auftraggeber der Studie hin. So wird in einem Beitrag für vhv Forum Wohneigentum, die Verbandszeitschrift des Bundesverbandes für Wohneigentum und Stadtentwicklung e. V., resümiert: »Die Studie Migranten-Milieus erweitert die bislang vorherrschende wohnungsmarktspezifische Betrachtungsweise von Migranten. Bei Analysen zur Situation dieses Perso-

\section{»Der Einfluss religiöser Traditionen wird weitgehend überschätzt «}

- Es wäre, zum Zweiten, bei aller angebrachten Skepsis zumindest überlegenswert, ob und inwieweit ein milieuorientierter Ansatz auch für Untersuchungen zum Verlauf von Integrationsprozessen fruchtbar gemacht werden kann.

- Zum Dritten liefert die Studie darüber hinaus potenziell Impulse für Überlegungen zur Entwicklung neuer und weiter differenzierter Integrationsmaßnahmen sowie von Ansprachewegen von Migrantinnen und Migranten. Versteht man Integrationsmaßnahmen und die Angebote sozialer Dienste als Produkte und deren Nutzer als Kunden, ließe sich beispielsweise erörtern, inwieweit in die Ausrichtung und Bewerbung solcher Maßnahmen und Angebote künftig auch die Berücksichtigung milieuspezifischer Präferenzen einfließen könnte.

Dies alles freilich unter der Prämisse, solche Angebote künftig auch unter Marketinggesichtspunkten betrachten zu wollen und zu können. Hierin liegt allerdings auch eine Begrenzung des Ansatzes. Schließlich lassen sich Integrationsmaßnahmen und sozialarbeiterische und sozialpädagogische Angebote und deren Nutzerinnen und Nutzer nicht simpel unter marktlogische Begründungszusammenhänge subsumieren. Sofern man Integrationsmaßnahmen gerade unter der Perspektive betrachtet, immer auch Unterstützungsangebote Sozialer Arbeit mit und für Migrantinnen und Migranten zu sein, entziehen sich diese nachgerade einer marktlogischen Monokausalität. nenkreises auf dem Wohnungsmarkt stehen bislang typischerweise sozial benachteiligte Gruppen im Vordergrund (Sozialhilfeempfänger, Bezieher unterer Einkommensgruppen, Personen mit geringer Formalbildung). Auf dem Wohnungsmarkt werden Migranten unter diesen Gegebenheiten als negativ konnotierte soziale Randgruppe betrachtet - zu Unrecht, wie die Analysen von Sinus Sociovision zeigen, denn diese sind in weiten Teilen auch eine durchaus attraktive Zielgruppe: zahlungskräftig und am Erwerb von Wohneigentum interessiert. «(Beck, Perry 2007: 192)

Was aber kein Argument dagegen ist, bestimmte Aspekte der Studie in die Entwicklung zukünftiger Maßnahmen und Angebote wenigstens mit einzubeziehen. Vor dem Hintergrund der Einschränkungen gilt es, die Marktforschungsstudie auf ihren möglichen Ertrag hinsichtlich zielgruppengerechterer Angebote und Beratungsstrukturen im Bereich der sozialen Arbeit zu sondieren - diese werden sich jedoch weniger an die Zahlungskräfigen richten.

Was abschließend noch zu formulieren bleibt, ist ein grundsätzlicher Einwand bezüglich der Bestimmung von Milieus in Abhängigkeit von der Dimension Migrationshintergrund in einer gesonderten Erhebung - und deren Implikationen. $\mathrm{Zu}$ fragen wäre beispielsweise, wieso die bisherigen klassischen Sinus-Mileu-Studien, deren Grundgesamtheit nach eigenem Bekunden die Wohnbevölkerung ab 14 Jahren in Deutschland bildet, also circa 64
Millionen Menschen, die Dimension Migrationshintergrund nicht exponierter berücksichtigten und damit zusammenhängende Items abgefragt haben.

Es wäre höchst interessant zu erfahren, inwieweit die bekannten "klassischen « Milieus mittlerweile über einen Migrationshintergrund verfügen und welche eben nicht - und wenn ja bzw. wenn nein, woran dies liegen könnte. Dies ließe durchaus Rückschlüsse auf die Durchlässigkeit von Milieugrenzen zu und auf sogenannte soziale Fahrstuhleffekte. Statt dessen wurde mit der vorgestellten Übertragung des Milieu-Ansatzes auf die Gruppe der Menschen mit Migrationshintergrund der Weg einer Sondererhebung beschritten, deren Ergebnis und deren Visualisierung nahelegen, es existierten quasi parallel $\mathrm{zu}$ den deutschen Milieus davon unabhängige migrantische Milieus. Die vorgelegte Studie verweist somit auf eine Erkenntnis- und Erhebungslücke der bisherigen Sinus-Milieus, die es bislang nicht vermochten, der Migrationsrealität in Deutschland ausreichend Rechnung zu tragen. Es ist deshalb zu begrüßen, dass das Thema Migration auch in der Welt der Milieustudien angekommen ist. Es steht indes aus, dieses in angemessener Weise einzubeziehen.

\section{Anmerkungen}

(1) Bei dem vorliegenden Artikel handelt es sich um die überarbeitete und ergänzte Fassung des Beitrages »Kartoffelgrafik jetzt auch mit Migrationshintergrund « aus der Zeitschrift " Migration und Soziale Arbeit", Heft $1 / 2008$.

(2) Weitere Ergebnisse siehe Internet: http://www.sinus-sociovision.de/ Download/Zentrale_Ergebnisse_ 16102007.pdf; Stand: 23.11.2007.

(3) Vgl. Internet http://www.sinus-sociovision.de

(4) Vgl. ebd. Internet http://www.sinussociovision.de/Download/Zentrale_Ergebnisse_16102007.pdf; Stand: 23. 11. 2007. Vgl. auch Beck, Sebastian/ Perry, Thomas 2007: "MigrantenMilieus. Erste Erkenntnisse über Lebenswelten und wohnungsmarktspezifische Präferenzen von Personen mit Migrationshintergrund in Deutschland " in: vhv Forum Wohneigentum 4 / Juli-September 2007, S. 187-195. 